\title{
Another light in the dark: Review of a new method for the arthroscopic repair of triangular fibrocartilage
} complex

Chris YK Tang, MBBS, B Fung, MD, Chan Rebecca, MSc HC (OT), CP Lung, MBBS

Purpose The triangular fibrocartilage complex (TFCC) is an anatomically and biomechanically important structure. Repair of radial-sided TFCC tear was used to be a challenge and not well-described in the literature. We designed a new method of radial-sided TFCC tear repair and found that it was also applicable for the ulnar-sided TFCC tear repair. The purpose of this study is to evaluate the functional outcome of new method of repair for TFCC tear.

Methods From October 2006 to December 2010, 10 patients underwent this operation were reviewed. There were 9 male and 1 female patients in the study with a mean age of 33.9 years old. The average post-operative follow-up period was 8 months. The results were graded according to the Mayo modified wrist score at the final follow-up.

Results In our study, by using the Mayo modified wrist score, 20\% ( 2 of the 10 patients) were rated as 'excellent', $30 \%$ (3 of the 10 patients) as 'good' and 50\% (5 of the 10 patients) as 'fair'. In the 5 patients rated as 'fair', they returned to regular employment or had restricted employment.

Conclusion Based on this small sample, we recommend this technique to be considered as an alternative method for TFCC repair.

Type of study/level of evidence Therapeutic III.

Key words triangular fibrocartilage complex, repair, arthroscopic 
$\mathrm{T}$ he triangular fibrocartilage complex (TFCC) was first described by Palmer. ${ }^{1}$ Anatomically, it consists of the articular disc, the dorsal and volar radioulnar ligaments, the meniscus homologue, the ulnar collateral ligament, and the sheath of the extensor carpi ulnaris. The TFCC of the wrist is supplied by small vessels that penetrate it in a radial fashion from the palmar, ulnar, and dorsal attachments of the joint capsule. The inner portion and the radial attachment have no blood vessels. ${ }^{2}$ This has a clinical significance that peripheral ulnar-sided TFCC tears are more likely to heal after repair.
Biomechanically, it functions as a cushion for ulnar carpus, ${ }^{3}$ stabilises the distal radioulnar joint (DRUJ) ${ }^{4-6}$ and stabilises ulnar carpus through ulnar carpal ligament complex. Untreated TFCC tear will cause arthritic changes in lunate, ulna and triquetrum, loss of motion and grip strength in the wrist. ${ }^{7}$

Palmer $^{8}$ classified TFCC tears as traumatic (type 1) and degenerative (type 2). Type 1 was subdivided according to the anatomical site of tissue disruption, whereas type 2 was based on the extent of degenerative process (Table 1).

\section{TABLE 1. Classification of TFCC tears}

Class of TFCC Tear

\begin{tabular}{ll}
\hline 1A & isolated central TFCC articular disk perforation \\
1B & peripheral ulnar-sided TFCC tear (with or without ulnar styloid fracture) \\
1C & distal TFCC disruption (disruption from distal ulnocarpal ligaments) \\
1D & radial TFCC disruption (with or without sigmoid notch fracture) \\
2A & TFCC wear \\
2B & TFCC wear with lunate and/or ulnar chondromalacia \\
2C & TFCC perforation with lunate and/or ulnar chondromalacia \\
2D & TFCC perforation with lunate and/or ulnar chondromalacia and with lunotriquetral \\
& ligament perforation \\
2E & TFCC perforation with lunate and/or ulnar chondromalacia and with lunotriquetral \\
& ligament perforation
\end{tabular}

As mentioned above, there was less vascularity in the radial side of TFCC, making repair less likely to heal than the ulnar side. ${ }^{2,9}$ Moreover, since radius has a relatively bigger diameter than ulna bone, it will be more difficult for the transosseous needle to target the radial insertion of TFCC from outside. This makes the radial-sided TFCC tear more difficult to be repaired by the outside-inside techniques. All the above reasons make radial-sided TFCC tear become a challenge to repair. As a result, management of radial-sided TFCC tear was only described in a few literatures. ${ }^{10-13}$ Long-term clinical improvement after repair of radial-sided TFCC tear has been reported in the 1990s. ${ }^{14}$ Since then, the repair of radial-sided TFCC tear began to gain more attention. Scott ${ }^{12}$ described a method for arthroscopic repair of radial-sided TFCC tear in 1994.

We used a new TFCC repair technique which has not yet been described in literatures for radial-sided TFCC tear. We found that this technique could also be applied to repair of ulnar-sided TFCC tear.

\section{PATIENTS AND METHODS}

A retrospective review was conducted for 11 adult patients treated by this new technique of TFCC repair, from October 2006 to December 2010. One patient was transferred to another hospital shortly after the operation due to geographical reason. As a result, 10 patients were included. 9 male and 1 female patients ranging from 20-58 years old, with a mean age of 33.9 years old, were evaluated. 7 patients injured the right hand and 3 patients injured the left hand. The dominant hand was involved in 7 cases. All of the cases were preceded by trauma to the wrist. The time from the accident to the operation had a mean of 8.3 months, ranging from 2 months to 24 months. Most of the presentations were wrist pain. Most patients had magnetic resonance arthrography (MRA) of the affected wrist before operation. The injection of contrast into the 
joint increased the sensitivity and specificity of TFCC tear detection, in comparison to magnetic resonance imaging of the wrist only.

The patients were reassessed after conservative treatment (which involves removable wrist splints and analgesic for pain control) for 2-3 months. All the patients in this study underwent operation due to persistent or worsening ulnar wrist pain, or significant power grip loss. The average post-operative follow-up was 8 months, ranging from 3 months to 13 months. At the final follow-up, the outcome was assessed by the Mayo modified wrist score.

\section{Surgical Technique}

The operations were performed under regional or general anesthesia with the patients in supine position. An upper arm tourniquet was applied with pressure 100 $\mathrm{mmHg}$ above systolic blood pressure. Finger traps were applied to the index, middle and ring fingers. The fingers are suspended with a $10 \mathrm{lb}$ traction by a sterile upright traction tower. 3-4 portal, 4-5 portal \& 6U portal were created. Wrist arthroscope was inserted for exploration to locate the TFCC tear. A probe was inserted via the 6R portal (for ulnar-sided TFCC tear), or the 4-5 portal (for radial-sided TFCC tear). The articular disk was then examined by the probe with the so-called trampoline test. Loss of tension and absence of disk rebound indicated there was detachment from the insertion sites. The $6 \mathrm{U}$ portal was used as an outflow.

For the repair of ulnar-sided TFCC tear, a meniscal-double-barrel cannula (Figure $1 \& 2$ ) was

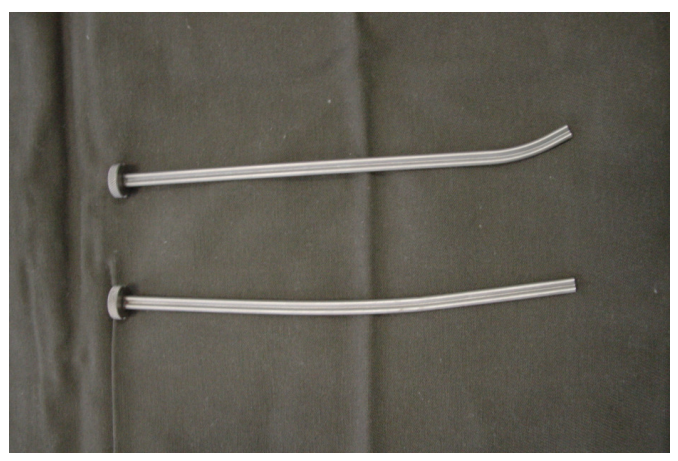

FIGURE 1: meniscal-double-barrel cannula

The cannula with curved tip is used for ulnar-sided TFCC tear repair inserted through the 4-5 portal, targeting the fovea. The curve of at the tip of the cannula also made the targeting of fovea simpler. The 3-4 portal was avoided because it was relatively more distant from the ulnar-sided TFCC. Insertion via this portal could cause iatrogenic cartilage injury more easily. $2 \mathrm{~K}$ wire drilled via the double barrel instrument, through the ulnar TFCC, distal ulna bone and exited on ulna aspect of ulna. A double-armed straight needle ( 2 straight needles sharing a common threaded suture) (Figure 3) was then employed. One of the needles was passed through the barrel, ulna bone, to the outside of skin. The other needle was then passed through another barrel, ulna bone, to the outside of skin (Figure 4). The 2 needles were then cut from the thread. A skin incision was made between the exit points of the 2 needles, and a knot was tied over the periosteum of ulna.

For the repair of radial-sided TFCC tear, A meniscal-double-barrel cannula was inserted through the $6 \mathrm{R}$ portal. $2 \mathrm{~K}$ wire drilled via the double barrel instrument, through the radial TFCC, distal radius and exited on radial aspect of radius. A double-armed straight needle (2 straight needles sharing a common threaded suture) was then employed. One of the needles was passed through the barrel, radius bone, to the outside of skin. The other needle was then passed through another barrel, radius bone, to the outside of skin (Figure 5). The 2 needles were then cut from the thread. A skin incision was made between the exit points of the 2 needles, and a knot was tied over the periosteum of radius.

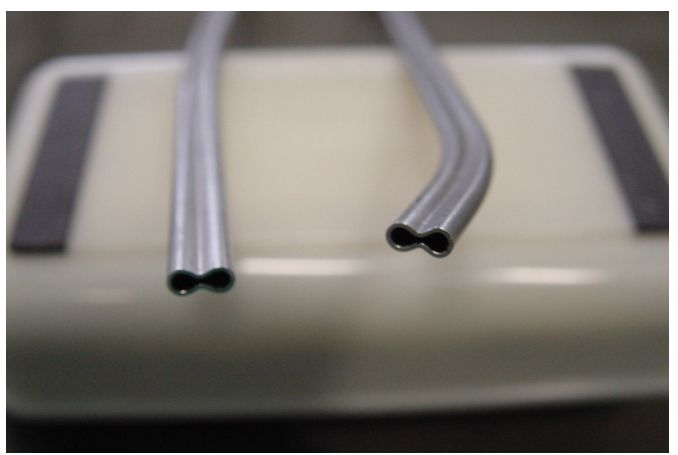

FIGURE 2: meniscal-double-barrel cannula (cross-section) 


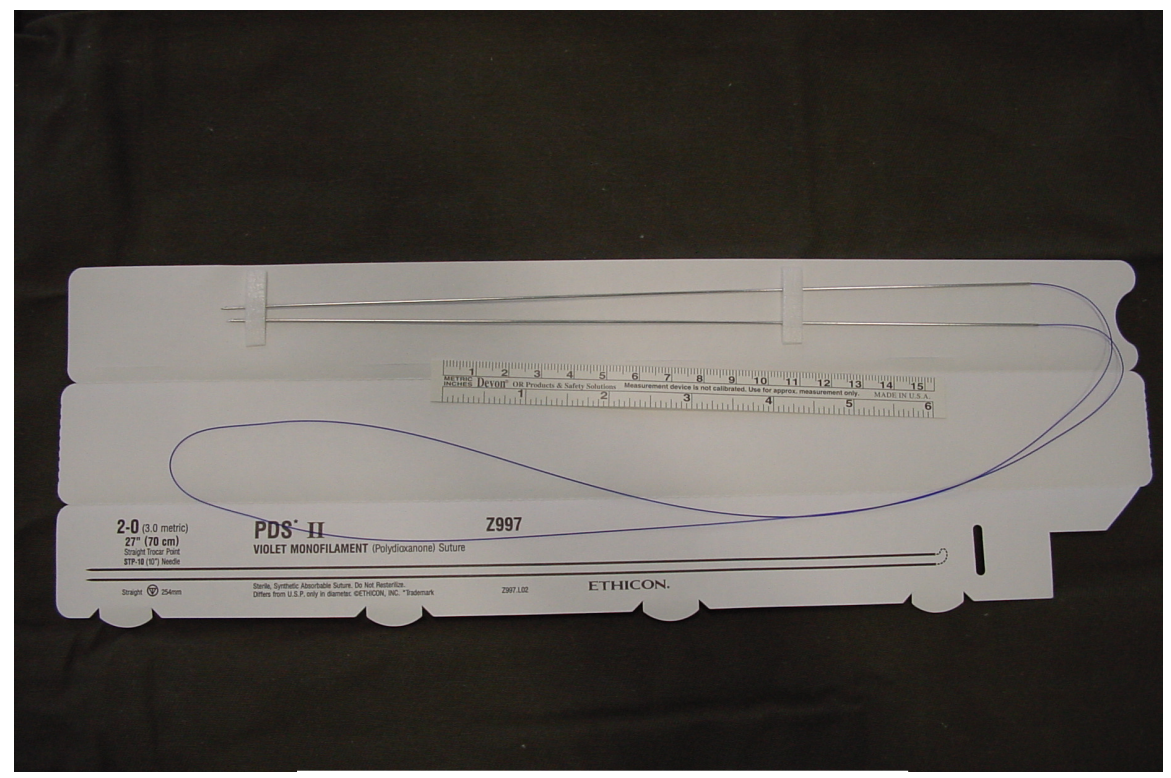

FIGURE 3: double-armed straight needle

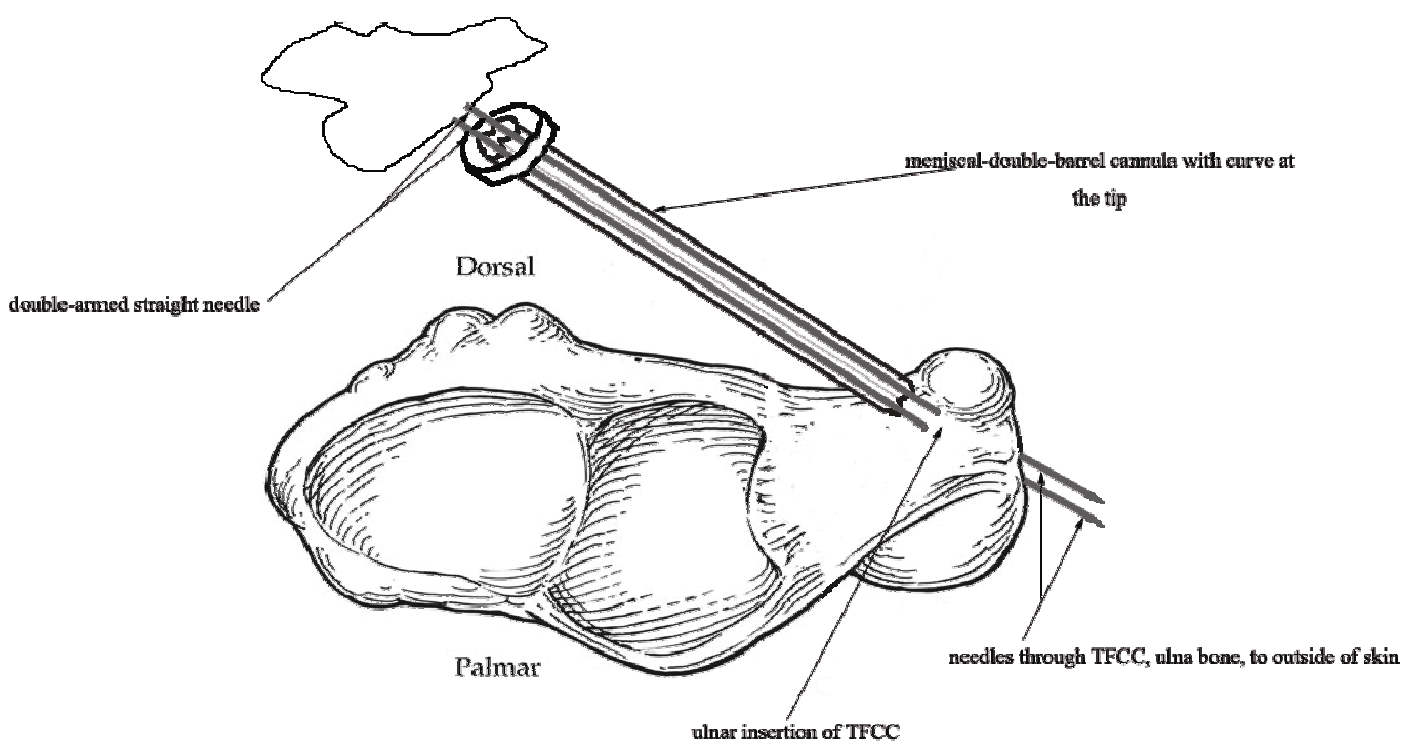

FIGURE 4: position of meniscal-double-barrel cannula in ulnar-sided TFCC tear repair (Modified from Carlsen B et al. Soft-tissue injuries associated with distal radius fratures. Operat Tech Orthop 2009; 19 (2): 107-18; with permission.) 


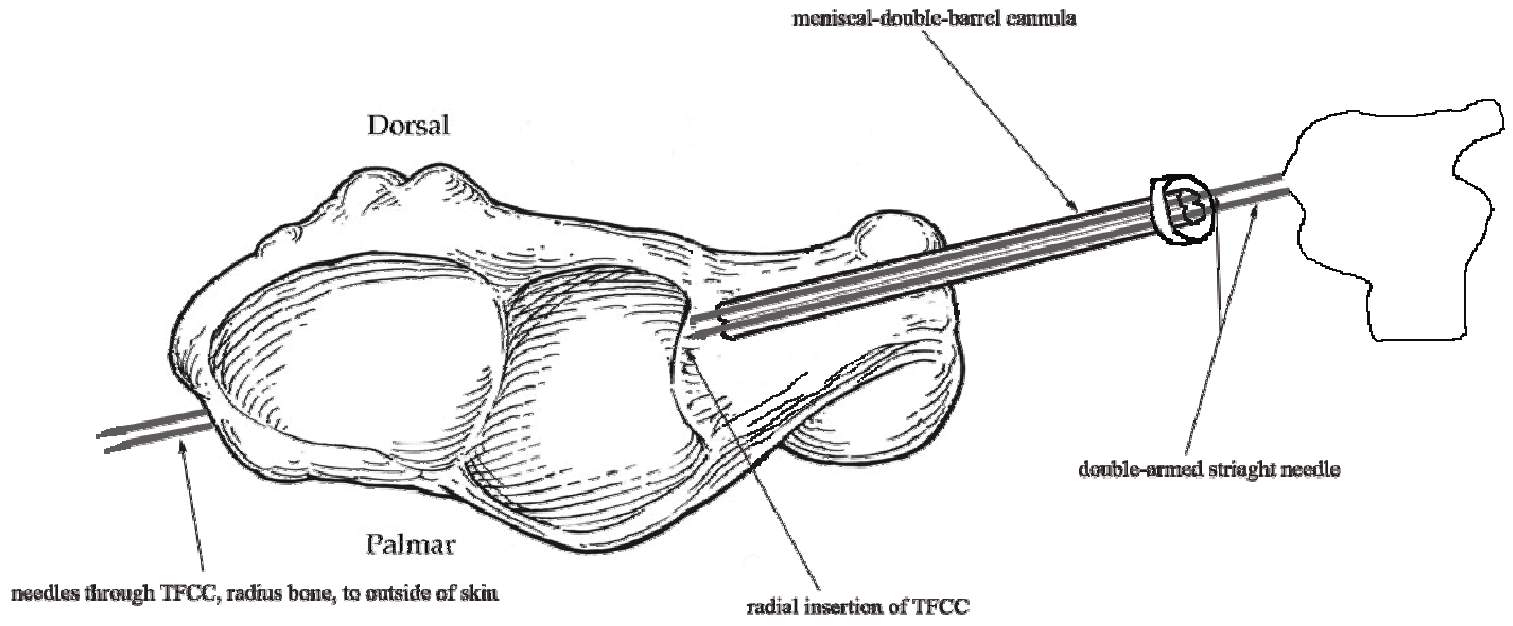

FIGURE 5: position of meniscal-double-barrel cannula in radial-sided TFCC tear repair (Modified from Carlsen B et al. Soft-tissue injuries associated with distal radius fratures. Operat Tech Orthop 2009; 19 (2): 107-18; with permission.)

\section{Postoperative Care}

Postoperatively, the wrist and forearm were immobilised at neutral position for 3 weeks by hinged elbow brace if the DRUJ was stable clinically before the operation. If the DRUJ was unstable clinically before the operation, the wrist and forearm were immobilised at supination for 3-4 weeks by hinged elbow brace. During the period of immobilization of wrist and forearm, there would be physiotherapy for fingers and shoulder mobilization.

After removal of brace, mobilization exercise and range of movement training were carried out. The patients underwent gradual strengthening exercise as pain tolerated, usually at 6 weeks post-operatively, followed by vocational rehabilitation.

\section{RESULTS}

The MRA findings in 7 of the 10 patients correlated well with the arthroscopic findings. In 1 patient, the initial MRA was normal, but the arthroscopic finding showed $30 \%$ of TFCC tear at sigmoid notch. The remaining 2 patients had the imaging done in private sector and the reports could not be traced.

On wrist arthroscopic examination, there were 3 cases of Palmer class 1A, 5 cases of class $1 \mathrm{~B}$ and 8 cases of class $1 \mathrm{D}$, in which there were 4 cases involved 2 or more sites of TFCC tear.

At the final follow-up, the outcome was assessed by the Mayo modified wrist score (Table 2). ${ }^{15} 20 \%$ (2 of the 10 patients) were rated as 'excellent', $30 \%$ (3 of the 10 patients) as 'good' and $50 \%$ (5 of the 10 patients) as 'fair'. Regarding the complications of the operation, 1 patient had radial and ulnar sided pain at entrance \& exit of $\mathrm{K}$ wire which was partially relieved by DRUJ band. He also had numbness over distribution of superficial branch of radial nerve which spontaneously resolved in 3 months. This was a known complication due to injury of the nerve at the exit of needle in radius. 


\begin{tabular}{|c|c|c|}
\hline Category & Description & Points \\
\hline \multirow[t]{4}{*}{ Pain } & No pain & 25 \\
\hline & Mild, occasional pain & 20 \\
\hline & Moderate, tolerable pain & 15 \\
\hline & Severe to untolerable pain & 0 \\
\hline \multirow[t]{4}{*}{ Functional status } & Return to regular employment & 25 \\
\hline & Restricted employment & 20 \\
\hline & Able to work, unemployed & 15 \\
\hline & Unable to work, pain & 0 \\
\hline \multirow[t]{6}{*}{ Motion (total motion) } & $120^{\circ}$ or more & 25 \\
\hline & $100^{\circ}-120^{\circ}$ & 20 \\
\hline & $90^{\circ}-100^{\circ}$ & 15 \\
\hline & $60^{\circ}-90^{\circ}$ & 10 \\
\hline & $30^{\circ}-60^{\circ}$ & 5 \\
\hline & $0^{\circ}-30^{\circ}$ & 0 \\
\hline \multirow[t]{5}{*}{ Grip strength } & $90 \%-100 \%$ & 25 \\
\hline & $75 \%-90 \%$ & 15 \\
\hline & $50 \%-75 \%$ & 10 \\
\hline & $25 \%-50 \%$ & 5 \\
\hline & $0 \%-25 \%$ & 0 \\
\hline
\end{tabular}

\section{DISCUSSION}

There are various methods of TFCC repair. For arthroscopic suturing of Palmer class 1B TFCC tear, the most common method is the outside-inside technique described by Zachee. ${ }^{16} 2$ sutures are introduced into the joint by a needle \& pulled out of the joint using forceps. A knot is tied into the sutures and drawn back into the joint. The other ends of sutures are then sutured subcutaneously with each other. Salvatore ${ }^{17}$ reported another method of arthroscopic suturing. Under arthroscopic guidance, edges of tear are debrided by a power shaver. A curved cannulated suture passer is then inserted through the floor of extensor carpi ulnaris sheath through the peripheral edge of the torn TFCC. A loop suture retriever is placed over the end of suture passer. A suture is passed through the passer. The suture retriever is then pulled back out of the joint, bringing the end of suture with it. The sutures are then tied down over the floor of the sixth extensor compartment.

Scott ${ }^{12}$ described a method similar to our method.
However, his method was only applied to repair of radial-sided TFCC tears. Also, he did not use the meniscal-double-barrel cannula. The meniscal-double-barrel cannula described in our method was used by our sport team for meniscal repair. By using this instrument, we could locate the site for $\mathrm{K}$-wire drill in a much shorter time, thus minimizing the operating time. In addition, our meniscal-double-barrel cannula may have a larger width compared to the smaller diameter of the cannula used by Scott. As a result, the distance between the exit points of the 2 needles can be greater and less number of sutures are needed.

In our experience, the above mentioned methods are time-consuming, even in experienced surgeons' hands. We designed a new method of TFCC repair. The advantage of this method is more user-friendly. The operating time ranged from 35 to 75 minutes (as performed by our senior author). Moreover, this method was relatively simple and straightforward. Once the 
meniscal-double-barrel cannula was placed in the right position, the rest of the operation became relatively easy. In our experience, junior trainees also had a shorter learning curve for this operation. In addition, our method aligned the torn TFCC better to their corresponding anatomical insertion. This was caused by the transosseous sutures which were not present in the outside-inside techniques. This resulted a better ligament to bone healing, and better restoration of function due to healing in the anatomical position. Despite the fact that Ruch ${ }^{18}$ had shown that there was no difference between transosseous and capsular repair of TFCC tears biochemically, the study was performed on cadaveric model and therefore had a few limitations. Firstly, it could not take post-operative immobilisationa and soft-tissue healing into account. Secondly, it did not apply to repair of chronic TFCC tear. Thirdly, the biomechanical study results were dependent on the No. 2.0 PDS (polydioxanone) sutures which were used in both groups. This caused an insignificant difference between the two techniques. While further studies should be performed, the authors still believed that restoring the insertion of TFCC to anatomical position would yield a better function of the TFCC.

The disadvantage of this method is that there may be iatrogenic injury to the cartilage by the insertion of meniscal-double-barrel cannula due to its relatively bigger size. For ulnar-sided TFCC tear repair, there would be risk for injuring lunate or cartilage of distal radius. With care, the incidence of cartilage damage can be avoided by stretching out the soft tissue at the entry portal. In our study, the wrist pain in the patients decreased after operation. The cartilage injury might be spontaneously healed, making it clinically insignificant. For radial-sided TFCC tear repair, there would be low risk for injuring the triquetrum because the space was roomy. This discrepancy of the operative risk made this method better for radial-sided TFCC tear. Also, similar to other inside-out techniques, there was a higher risk of injuring the superficial cutaneous nerve during the passage of the meniscal needles.

In our study, most patients underwent MRA before the operation. 8 patients had results of pre-operative MRA of the affected wrist available. 7 of them $(87.5 \%)$ correlated well with the arthroscopic result. Our study showed that MRA had a high sensitivity $(87.5 \%)$, but less site-specific when compared to wrist arthroscopy (2 out of 8 patients (25\%) had another site of tear in arthroscopy in contrast to MRA). These are all consistent with previous studies ${ }^{19}$, ${ }^{20}$, showing MRA has a high positive predictive value $(0.95)$ but a low negative predictive value $(0.50){ }^{20}$. The high sensitivity of MRA suggests that it can be done as a routine to evaluate TFCC injury. However, in patients with clinical suspicion of TFCC tear, the negative results of MRA should be interpreted with caution.

5 patients in our study had fair scores after the operation. These were mainly contributed by the relatively less satisfactory grip strengths in the Mayo modified wrist score. All of them only had occasional mild wrist pain, the lower grip strengths therefore could not be explained as a complication of the operation. The authors believed that these relatively less satisfactory grip strengths were due to inadequate post-operative training. Functionally, they returned to regular employment or had restricted employment.

In conclusion, this new method has a good clinical outcome based on this small sample and we recommend this technique as an alternative for TFCC repair because of its advantages.

\section{REFERENCES}

1. Palmer AK, Werner FW. The triangular fibrocartilage complex of the wrist--anatomy and function. J Hand Surg Am. 1981 Mar;6(2):153-62.

2. M. S. Bednar, S. P. Arnoczky, A. J. Weiland. The microvasculature of the triangular fibrocartilage complex: its clinical significance. J Hand Surg Am. 1991;16(6):1101-5.

3. Hara T, Horii E, An KN, Cooney WP, Linscheid RL, Chao EY. Force distribution across wrist joint: application of pressure-sensitive conductive rubber. J Hand Surg Am. 1992 Mar;17(2):339-47.

4. Stuart PR, Berger RA, Linscheid RL, An KN. The dorsopalmar stability of the distal radioulnar joint. J Hand Surg Am. 2000 Jul;25(4):689-99.

5. H. Kihara, W. H. Short, F. W. Werner, M. D. Fortino, A. K. Palmer. The stabilizing mechanism of the distal radioulnar joint during pronation and supination. J Hand Surg Am. 1995;20(6):930-6.

6. J. R. Haugstvedt, R. A. Berger, T. Nakamura, P. Neale, L. Berglund, K. N. An. Relative contributions of the ulnar attachments of the triangular fibrocartilage complex to the dynamic stability of the distal radioulnar joint. J Hand Surg Am. 2006;31(3):445-51.

7. J. T. Shih, Y. T. Hou, H. M. Lee, C. M. Tan. Chronic triangular fibrocartilage complex tears with distal radioulna joint instability: A new method of triangular fibrocartilage complex reconstruction. J Orthop Surg (Hong Kong). 2000;8(1):1-8.

8. A. K. Palmer. Triangular fibrocartilage complex lesions: a classification. J Hand Surg Am. 1989;14(4):594-606.

9. R. G. Thiru, D. C. Ferlic, M. L. Clayton, D. C. McClure. Arterial anatomy of the triangular fibrocartilage of the wrist and its surgical significance. J Hand Surg Am. 1986;11(2):258-63.

10. A. Minami, K. Kaneda, H. Itoga. Hemiresection-interposition arthroplasty of the distal radioulnar joint associated with repair of triangular fibrocartilage complex lesions. J Hand Surg Am. 1991;16(6):1120-5. 
11. J. D. Hermansdorfer, W. B. Kleinman. Management of chronic peripheral tears of the triangular fibrocartilage complex. J Hand Surg Am. 1991;16(2):340-6.

12. S. D. Sagerman, W. Short. Arthroscopic repair of radial-sided triangular fibrocartilage complex tears. Arthroscopy. 1996;12(3):339-42.

13. W. P. Cooney, R. L. Linscheid, J. H. Dobyns. Triangular fibrocartilage tears. J Hand Surg Am. 1994;19(1):143-54.

14. Cooney WP, Sagerman SD, W. H. Short. Arthroscopic repair of the radial sided TFCC tears: A follow-up study. Proceedings of the 49th Annual Meeting of the American Society of Surgery of the Hand, Cincinnati, OH. 1994;19:143-154.

15. P. C. Amadio, T. H. Berquist, D. K. Smith, D. M. Ilstrup, W. P. Cooney, 3rd, R. L. Linscheid. Scaphoid malunion. J Hand Surg Am. 1989;14(4):679-87.

16. B. Zachee, L. De Smet, G. Fabry. Arthroscopic suturing of TFCC lesions. Arthroscopy. 1993;9(2):242-3.
17. S. J. Corso, F. H. Savoie, W. B. Geissler, T. L. Whipple, W. Jiminez, N. Jenkins. Arthroscopic repair of peripheral avulsions of the triangular fibrocartilage complex of the wrist: a multicenter study. Arthroscopy. 1997;13(1):78-84.

18. D. S. Ruch, S. R. Anderson, M. R. Ritter. Biomechanical comparison of transosseous and capsular repair of peripheral triangular fibrocartilage tears. Arthroscopy. 2003;19(4):391-6.

19. L. Pederzini, R. Luchetti, O. Soragni, M. Alfarano, G. Montagna, E. Cerofolini, R. Colombini, J. Roth. Evaluation of the triangular fibrocartilage complex tears by arthroscopy, arthrography, and magnetic resonance imaging. Arthroscopy. 1992;8(2):191-7.

20. S. Joshy, S. Ghosh, K. Lee, S. C. Deshmukh. Accuracy of direct magnetic resonance arthrography in the diagnosis of triangular fibrocartilage complex tears of the wrist. Int Orthop. 2008;32(2):251-3. 